Cell Research (2002); 12(3-4):207-214

http://www.cell-research.com

\title{
The distribution of cofilin and DNase I in vivo
}

\author{
Deepak CHHABRA*, Shisan BAO, CRistobal G dos REMEDiOS \\ Muscle Research Unit, Department of Anatomy and Histology, Department of Pathology, Institute for Biomedical \\ Research, F13, University of Sydney, NSW 2006, Australia
}

\begin{abstract}
Actin is the principal component of the cytoskeleton, a structure that can be disassembled and reassembled in a matter of seconds in vivo. The state of assembly of actin in vivo is primarily regulated by one or more actin binding proteins (ABPs). Typically, the actions of ABPs have been studied one by one, however, we propose that multiple ABPs, acting cooperatively, may be involved in the control of actin filament length. Cofilin and DNase I are two ABPs that have previously been demonstrated to form a ternary complex with actin in vitro. This is the first report to demonstrate their co-localisation in vivo, and differences in their distributions. Our observations strongly suggest a physiological role for higher order complexes of actin in regulation of cytoskeletal assembly during processes such as cell division.
\end{abstract}

Key words: actin, cofilin, DNase I, actin-binding protein, ternary complex.

\section{INTRODUCTION}

Actin $(42 \mathrm{kDa})$ is the principal component of the cytoskeleton. It consists of globular monomers (Gactin) that polymerise to form helically symmetrical filaments (F-actin). A dynamic cytoskeleton is important in numerous cellular activities ranging from maintenance of cell shape to phagocytosis and cytokinesis. This reversible assembly of actin is regulated by a large number of actin-binding proteins (ABPs) and with few exceptions, these have been studied individually rather than in combinations. ABPs influence the state of actin assembly and/or maintain the monomer pool above its critical concentration[1].

Binding an $\mathrm{ABP}$ may influence the interactions of actin with other ligands. For example the exchange

\footnotetext{
* Corresponding author: Mr. Deepak Chhabra Muscle Research Unit, Department of Anatomy and Histology, Institute for Bio medical Research, F13, University of Sydney, Sydney, NSW 2006, Australia Tel: +61 29351 3266, Fax: +61 293512813 , E-mail: dchhabra@anatomy.usyd.edu.au Received May-20-2002 Revised July-16-2002 Accepted July-222002
}

of the bound nucleotide of actin is promoted by profilin[2] and inhibited by cofilin[3] despite the fact that these ABPs bind to a nearly identical loci on actin[4]. Furthermore, cofilin allosterically inhibits the binding of phalloidin[5] and tropomyosin[6] suggesting that $\mathrm{ABPs}$ may also modulate the binding of other ABPs. This would allow finer control of actin assembly and facilitate subtle remodelling of the cytoskeleton.

Cofilin $(19 \mathrm{kDa})$ is a principal player in regulating the dynamics of actin. Like most ABPs, cofilin binds to the barbed end of actin (subdomains 1 and 3 ). It is capable of regulating the average length of actin filaments by severing[7] or depolymerising in a $\mathrm{pH}$ sensitive manner[8]. The biological action of cofilin is regulated via phosphorylation[9] by LIMkinases[10] and by the binding of phosphoinositide lipids[11]. In vivo cofilin is involved in cell division, actin treadmilling and recycling of older actin filaments.

DNase I ( $31 \mathrm{kDa})$ is a secreted enzyme involved in digestion of DNA and has been implicated in apoptosis[12]. Although not widely recognised as an 
ABP, Lazarides and Lindberg [13] were the first to suggest that DNase I may be a cytoskeletal protein. They proposed three possible biological roles: (1) actin controls nucleotide hydrolysis of DNase I during the cell cycle; (2) the actin-DNase I complex has a specific function in DNA metabolism; and (3) the primary function of DNase I is related to the formation and function of actin filaments rather than the degradation of DNA. The fact that phosphoinositides can dissociate DNase I from its complex with monomeric actin[11] also suggests this interaction may be biologically relevant.

DNase I binds stronger to monomeric actin ( Kd in the $\mathrm{n} M$ range) than to $\mathrm{F}$-actin $\left(\mathrm{K}_{\mathrm{d}}=100 \mu M\right)$ where it acts as a capping protein and increases the dissociation of subunits from the pointed ends of filaments[14]. Thus, unless the concentration of DNase $\mathrm{I}$ is high, it is unlikely to bind to actin filaments. In vivo, the enzymatic activity of DNase I is inhibited by the binding of G-actin. However unlike most ABPs, including cofilin, it binds to the region of actin oriented towards the pointed end of F-actin, specifically to subdomain 2 (the DNase I binding loop) and to subdomain 4 (Fig 1)[15].

We have previously reported that it is possible for both cofilin and DNase I to bind simultaneously to G-actin in vitro forming a cofilin-actin-DNase I ternary complex[16]. Furthermore, we have demonstrated that the binding between cofilin, actin and DNase I in the ternary complex in vitro is essentially $\left(\mathrm{Ca}^{2+}\right.$ or $\left.\mathrm{Mg}^{2+}\right)$ [17].Conversely, the cofilin-actin and actin-DNase I binary complexes are strongly influenced by buffer nucleotides and cations. The formation of the cofilin-actin-DNase I ternary complex has since been shown to be a cooperative process [18].

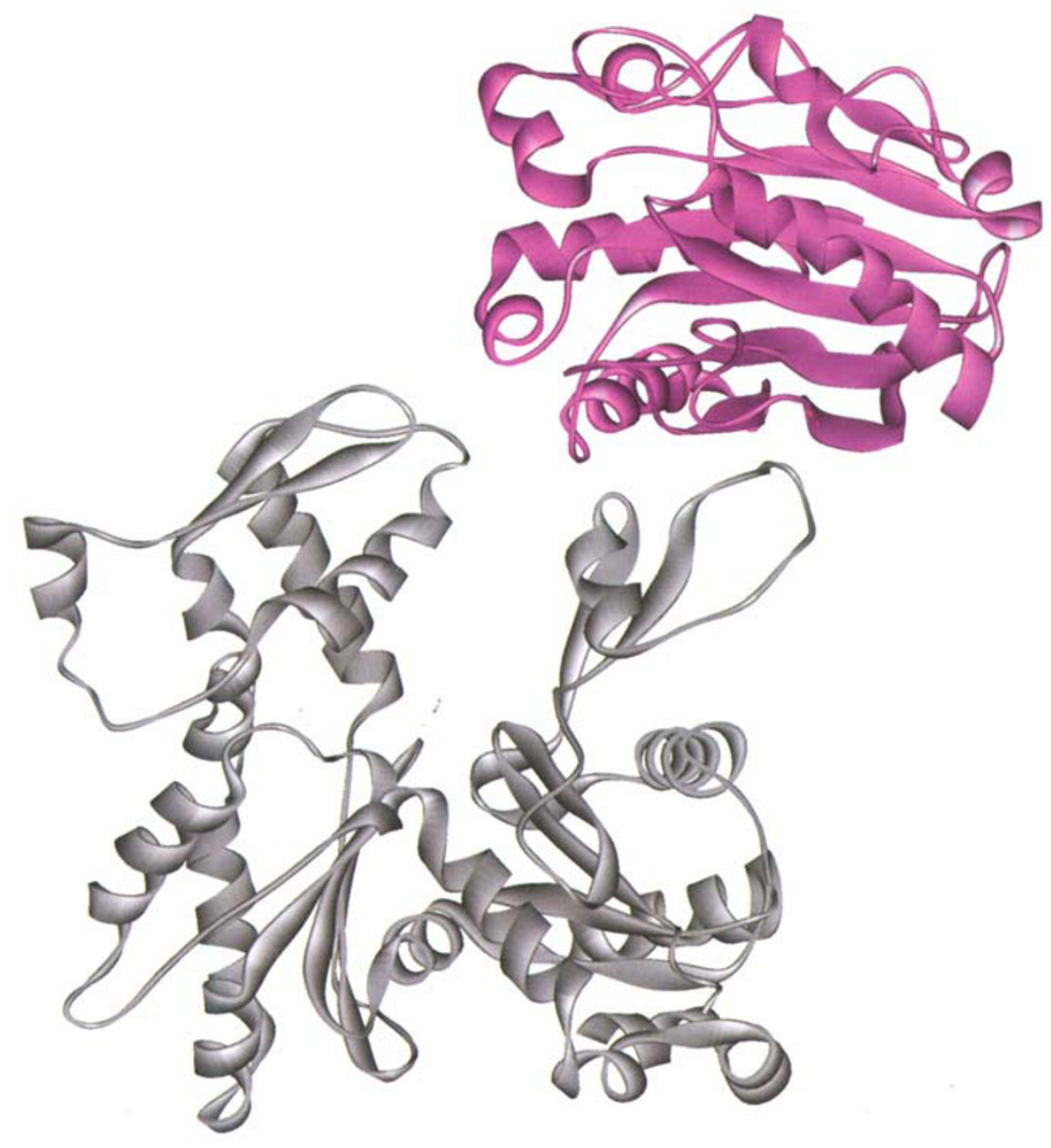

Fig 1. Crystal structure of actin-DNase I complex (PDB accession number, 1ATN)

DNase I (purple) interacts with loop on subdomain 2 of actin (grey)[15]. The underside of actin in this figure contains the binding site of a number of ABPs including cofilin. 
A crucial factor in establishing the biological relevance of DNase I is the development of proof that the ternary complex actually occurs in vivo.

\section{MATERIALS AND METHODS}

\section{Actin preparation}

Actin was prepared from an acetone-dried powder of rabbit skeletal muscle according to the method of Spudich and Watt[19] with slight modifications as described in Barden and dos Remedios [20]. G-actin was snap frozen in liquid nitrogen in G buffer $(2 \mathrm{mM}$ Tris $\mathrm{pH}$ 8.0, $0.2 \mathrm{mM}$ ATP, $0.1 \mathrm{mM} \mathrm{CaCl}{ }_{2}$ ) then freeze-dried and stored at $-20^{\circ} \mathrm{C}$. Monomeric actin concentration was determined from its OD290, where $\mathrm{E}^{0.1 \%}=0.63 \mathrm{~cm}-1[21]$.

\section{Expression and purification of chicken cofilin}

Recombinant chick embryonic skeletal muscle cofilin was expressed as a glutathione- S-transferase (GST) fusion protein. The clone for this fusion protein was a gift from Dr Takashi Obinata. Briefly, E. Coli transformants were grown at $37^{\circ} \mathrm{C}$ in $\mathrm{LB}$ medium (Gibco) containing $60 \mathrm{mg} / \mathrm{mL}$ ampicillin and induced with $1 \mathrm{~m} M$ isopropyl- $\beta$-D-thiogalactopyranoside when the cells were in log phase of growth $(\mathrm{OD} 600 \approx 0.6 / \mathrm{cm})$. After $3 \mathrm{~h}$ growth, the cells were harvested by centrifugation and disrupted using a French press. After centrifugation, the supernatant was applied to a glutathione Sepharose 4B column (Pharmacia, Piscataway, NJ, USA) equilibrated with phosphate buffered saline (PBS, 0.35 M) NaCl, $2 \mathrm{mM} \mathrm{KCl}, 10 \mathrm{~m} M \mathrm{Na}_{2} \mathrm{HPO}_{4}, 2 \mathrm{mM} \mathrm{KH_{2 }} \mathrm{PO}_{4}, \mathrm{pH}$ 7.2) containing $0.5 \mathrm{mM}$ PMSF and $1 \mathrm{mM}$ EDTA. The column was washed with 2 vol of thrombin buffer ( $50 \mathrm{mM}$ Tris $\mathrm{pH}$ 8.0, $2.5 \mathrm{mM}$ $\mathrm{CaCl}_{2}$ and $50 \mathrm{mM} \mathrm{NaCl}$ ). The matrix was suspended in $100 \mathrm{U}$ of thrombin/L of culture and incubated at $37^{\circ} \mathrm{C}$ for $1 \mathrm{~h}$ with the matrix being mixed at 15 min intervals. Cofilin was eluted with $2-$ 3 vol of thrombin buffer. The purified cofilin was dialysed overnight against $10 \mathrm{mM}$ Pipes ( $\mathrm{pH}$ 6.8) and further purified by application to a Mono-S-cation exchange column and eluted with a 0-0.5 $\mathrm{M}$ gradient of $\mathrm{NaCl}$. The protein concentration was determined from the $\mathrm{OD}_{280}$, where $\mathrm{E}^{0.1 \%}=0.93 \mathrm{~cm}^{-1}[22]$.

\section{DNase I}

DNase I (DPRF grade) was purchased from Worthington Biochemicals (Freehold, NJ, USA) and used without further purification. DNase I protein concentrations were determined from the OD280, where $\mathrm{E}^{0.1 \%}=1.1 \mathrm{~cm}^{-1}[23]$.

\section{Native polyacrylamide gel electrophoresis}

An actin-DNase I complex was formed by incubation of DNase I $(2.5 \mu M)$ with excess actin $(5.0 \mu M)$. Increasing concentrations of cofilin $(0.7,1.4,2.1,2.8,4.2$ and $7.0 \mu M)$ were then titrated into this actin-DNase I -actin solution. Samples were taken and analysed using native (non-denaturing) polyacrylamide gel electrophoresis (PAGE).

Native PAGE of samples was performed using a BioRad MiniPROTEAN $^{\circledR}$ II system (Richmond, CA, USA) and a discontinuous
Tris-glycine buffer system[24] with omission of SDS from all solutions. The stacking gel comprised $4 \%$ acrylamide in $80 \mathrm{mM}$ Tris-glycine ( $\mathrm{pH}$ 6.8) and the separating gel comprised $10 \%$ acrylamide in the same buffer adjusted to $\mathrm{pH}$ 8.8. Running buffer (25 mM Tris pH 8.3, $192 \mathrm{~m} M$ glycine, $0.2 \mathrm{~m} M$ ATP, $0.2 \mathrm{~m} M \mathrm{CaCl}_{2}$ ). Samples were run at $120 \mathrm{~V}$ for $90 \mathrm{~min}$ at room temperature.

Since these gels were performed under non-denaturing conditions, proteins migrate according to their charge-density ratio rather than their apparent molecular weights as they do in SDS-PAGE gels. Non-covalent interactions between proteins are not disrupted under these conditions.

Gels were stained with $1 \%(\mathrm{w} / \mathrm{v})$ Coomassie Brilliant Blue R250 in $40 \%(\mathrm{v} / \mathrm{v})$ ethanol, $7 \%(\mathrm{v} / \mathrm{v})$ acetic acid and destained in $40 \%$ $(\mathrm{v} / \mathrm{v})$ ethanol and $7 \%(\mathrm{v} / \mathrm{v})$ acetic acid.

Gels were scanned using a Molecular Dynamics scanning densitometer (Sunnyvale, CA, USA.) and Coomassie-stained protein bands were quantified by Image Quant 5.1 software (Molecular Dynamics Inc; Sunnyvale, CA, USA).

\section{Distribution of cofilin and DNase I in vivo}

The localisation of DNase I and cofilin proteins was examined by immunofluorescent labelling and confocal microscopy. Virulent monkey kidney epithelial cells (ACTC no. CRL1586) were suspended in $10 \%$ foetal calf serum (FCS) in RPMI-1640 medium (Sigma-Aldrich; St. Louis, MO, USA) and incubated overnight in a $35 \mathrm{ml}$ culture flask under standard culture conditions (SCC, 37 ${ }^{\circ} \mathrm{C}$ in a humidified chamber atmosphere of $5 \% \mathrm{CO}_{2}$ and air). Following incubation, the supernatant was removed and the cells were washed three times in PBS and resuspended in T/E solution $(0.5 \% \mathrm{w} / \mathrm{v}$ trypsin, I mM EDTA). The cells were centrifuged at $4000 \times \mathrm{g}$ for $5 \mathrm{~min}$ and the pellet was washed in PBS and resuspended in $10 \%$ FCS in RPMI-1640 to a final density of $10^{6}$ cells $/ \mathrm{ml}$. Cells were subsequently seeded on $18 \mathrm{~mm}$ glass coverslips and incubated overnight under SCC. Cells were then washed three times in PBS, fixed in 3\% paraformaldehyde in PBS for 20 min at room temperature and permeabilised in cold acetone at $-20^{\circ} \mathrm{C}$ for $5 \mathrm{~min}$. Cells were then washed and incubated in 10\% FCS in RPMI-1640 for 45 minutes for blocking.

DNase I was labelled by incubating with a 1 in 250 dilution of rabbit anti-DNase I antibody (Sigma-Aldrich, St. Louis, MO, USA) for $45 \mathrm{~min}$ proceeded by a 1 in 200 dilution of a sheep anti-rabbit antibody conjugated with FITC (SAR-FITC) for $1 \mathrm{~h}$. Cofilin was labelled by incubating with a 1 in 200 dilution of rabbit anti-cofilin antibody (Cytoskeleton, Denver, CO, USA) for 45 min proceeded by a 1 in 200 dilution of a donkey anti-rabbit antibody-biotin conjugate (DAR-B) for $30 \mathrm{~min}$ and a 1 in 200 dilution of streptavidin-Cy 3 conjugate for $30 \mathrm{~min}$. Cells were washed and blocked in RPMI-1640 supplemented with $10 \%$ FCS for 45 min between each step. To eliminate cross-reactivity between the secondary antibodies, excess SAR-FITC was incubated for $1 \mathrm{~h}$ at 37 ${ }^{\circ} \mathrm{C}$ to saturate all the binding sites on the DNase I primary antibody. Subsequently, rabbit anti-cofilin antibody was incubated and DARB labelling was applied. Omission of either primary antibody or replacement with an isotype control antibody resulted in abolishment of yellow co-localisation regions (data not shown) support- 
ing the assumption that our antibody staining is specific for the corresponding antigen. The specificity of the antibody labelling is also consistent with the different distributions of the two ABPs.

The cells were viewed under a Leica TCS NT confocal microscope (Heidelberg, Germany) with bandpass filters centred at 525 $\mathrm{nm}$ and $590 \mathrm{~nm}$ in order to spectrally isolate the FITC and Cy3 probes respectively. Approximately $1 \mathrm{~mm}$ optical sections were taken in the vertical axis. Layered images were analysed using Adobe Photoshop 6.0 (Adobe Systems Japan; Tokyo, Japan) to demonstrate the simultaneous distribution of cofilin and DNase I and the distribution of co-localised cofilin and DNase I. The amount of labelled protein and co-localised molecules was quantified by Image Pro-Plus 4.5 (Scitech, Vic, Australia).

\section{RESULTS}

\section{Native polyacrylamide gel electrophoresis}

Fig 2 shows a Coomassie-stained native polyacrylamide gel of cofilin titrated into a mixture of actinDNase I $(2.5 \mu M)$ and free actin $(2.5 \mu M)$ annotated by the volume densities of each band below. The concentration of cofilin in each lane (numbered 1-7 below the gel) is indicated above the respective lane. The identities of protein bands are shown adjacent to the relevant band.

Lanes 2-4 demonstrates the preferential binding of cofilin to actin-DNase complex rather than to free actin. Addition of increasing concentrations of cofilin to a mixture of actin-DNase I complex and free actin results in increasing quantities of cofilinactin-DNase I ternary complex coupled to decrease in the volume density of the actin-DNase I band. Conversely, the volume density of actin remains approximately constant. In lanes 5-7 no actin-DNase I complex is present because it has been converted to cofilin-actin-DNase I ternary complex. At this point cofilin binds to free actin, with increased volume density of cofilin-actin band coupled to a decreased volume density of the free actin.

\section{Distribution of cofilin and DNase I in vivo}

Fig 3A shows a phase-contrast presentation of a representative quiescent (above) and dividing cell (below). There is a prominent cleavage furrow within the dividing cell. The nuclei are identified as "empty" regions within the cells (upper border of the quiescent cell; binucleated nucleus in the dividing cell).

Fig 3B illustrates the distribution of the FITC probe, thereby demonstrating the distribution of intracellular DNase I (green). DNase I is seen diffusely throughout the cells but is greatly reduced or absent from the regions corresponding to the nuclei. The distribution in the cytoplasm is discrete rather than evenly dispersed and there appears to be no preferential localisation of DNase I in any specific region of the cytoplasm.

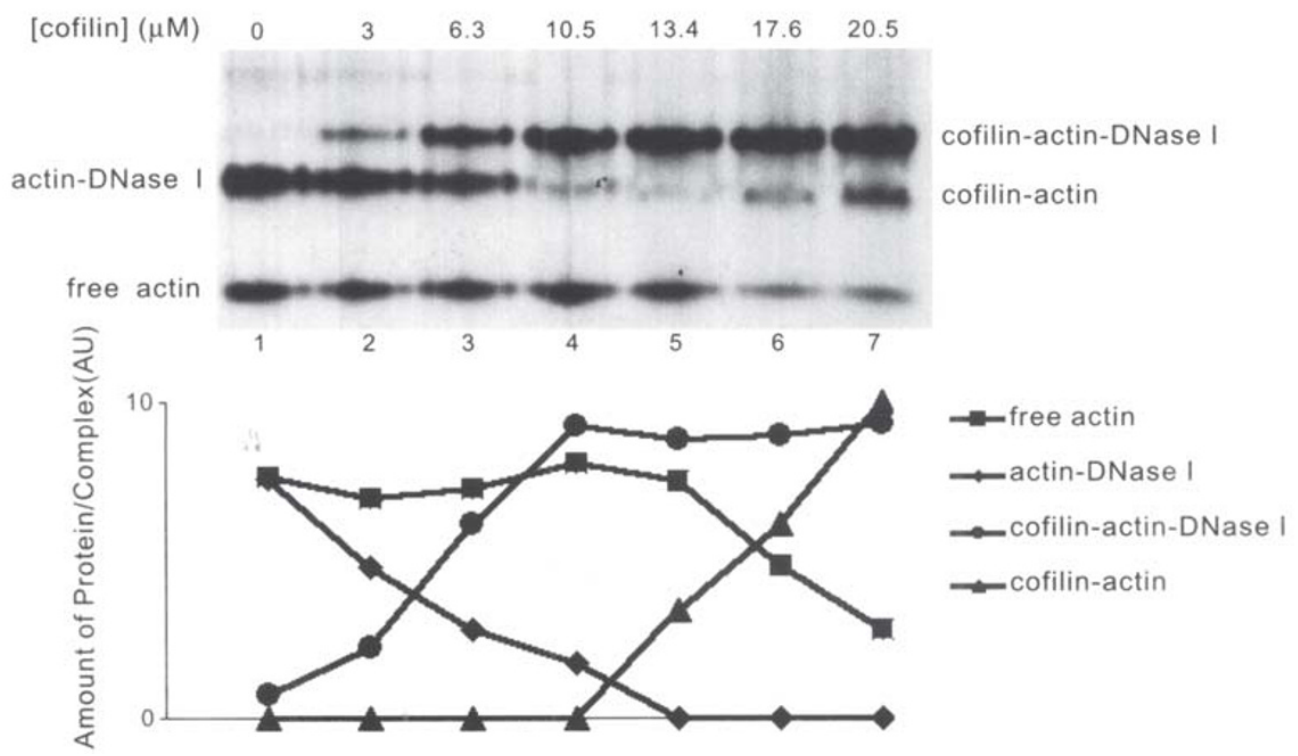

Fig 2. A native polyacrylamide gel shows the effect of titrating cofilin into a mixture of actin-DNase I complex and free actin. Lanes 1-7 contain a mixture of $8 \mu \mathrm{M}$ DNase I and $14 \mu \mathrm{M}$ actin and increasing concentrations of cofilin $(0,0.7,1.4,2.1,2.8,4.2$ and $7.0 \mu \mathrm{M}$ respectively). 

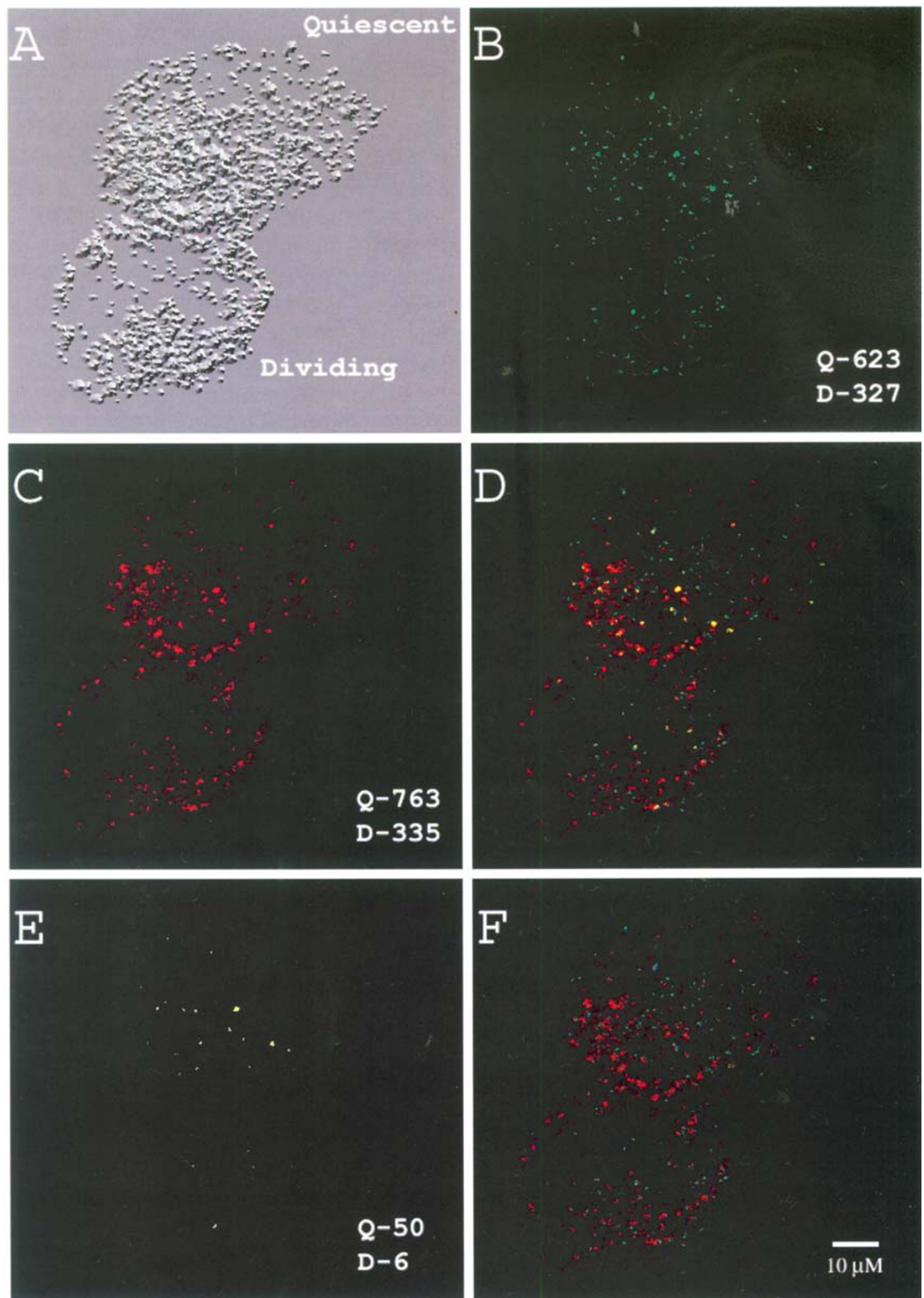

Fig 3. Distributions of intracellular DNase I (green) and cofilin (red) seen under a confocal microscope. The FITC and Cy3 probes were spectrally isolated by setting bandpass filters at $525 \mathrm{~nm}$ and $590 \mathrm{~nm}$ respectively. (A) Phase contrast image of representative cells (B) distribution of DNase I only; (C) distribution of cofilin only; (D) simultaneous visualisation of cofilin and DNase I; (E) distribution of co-localised cofilin and DNase I, and; (F) distribution of cofilin and DNase I not associated with each other. The number of labelled protein is quantified in image units for the quiescent (Q) and dividing cell (D). 
Fig $3 \mathrm{C}$ illustrates the distribution of the $\mathrm{Cy} 3$ probe, thereby demonstrating the distribution of intracellular cofilin (red). It appears to preferentially aggregate at the periphery of the dividing cell. It also appears to be concentrated at the junction between the upper cell and lower dividing cell. There is a marked decrease in its nuclear localisation.

Fig 3D shows the overlapped signals from the FITC and Cy 3. Regions of co- localisation of these two probes appear as yellow spots. The co-localised signal has been optically isolated and are displayed in Figure 3E. This suggests there is a co- distribution of a cofilin-actin-DNase I ternary complex (yellow). The yellow spots appear to be co-incident with the largest cofilin aggregates. No overlap is seen in the regions corresponding to the nuclei, nor is there significant overlap in the cortical regions corresponding to high concentrations of cofilin. For comparison, the signals from probes that do not overlap are displayed in Fig 3F.

\section{DISCUSSION}

Regulation of the cytoskeleton is complex and it is likely that it requires more than one $\mathrm{ABP}$ to regulate actin assembly. Formation of a cofilin-actinDNase I ternary complex has been demonstrated in vitro using both native (non-denaturing) PAGE gels [17] and Phast gels[18]. These reports determined that cofilin and DNase I bind cooperatively to actin forming a stable complex that is fairly insensitive to buffer conditions. The cooperative binding of cofilin to actin-DNase I demonstrated by Nosworthy et al has been confirmed quantitatively in this report. However this is the first report to provide evidence for a simultaneous distribution of cofilin and DNase I with actin in vivo. It is interesting to note that although the ratio of labelled cofilin to DNase I is approximately 1 , their distributions differ markedly.

Cofilin has previously been reported as being diffusely distributed throughout the cell, with increased localisation at regions where the cytoskeleton is highly dynamic. This includes the leading edge of Lamellipodia[25] and the cleavage furrow in dividing cells[26], [27]. These previous findings are consistent with our results. We found cofilin localised near the cell periphery in the dividing cell and scattered throughout the cytoplasm but excluded from the nucleus. In vivo, cofilin functions to rapidly dis- assemble actin near the cell periphery facilitating changes in cell shape during cell division. Cofilin binds to ADP-actin subunits in F-actin and promotes dissociation at the pointed ends of these filaments deeper in the cortex. They remain complexed to ADPactin monomers until the actin-bound nucleotide is exchanged for ATP under the influence of profilin.

The presence of cofilin near the border between associated cells may be explained by its role in cytokinesis from which these two cells formed. The cleavage furrow appears within a dividing cell during cytokinesis as a result of the constriction of the contractile ring. This contractile ring is formed by a bundle of approximately 20 actin filaments, which gradually constrict by up to $90 \%$ [28]. Although the actin filaments at the cleavage furrow gradually shorten, the total number of filaments remains constant. Cofilin severs actin and is involved in bringing about the gradual constriction of the F-actin contractile ring at the cleavage furrow.

DNase I appears to be expressed in low concentrations and distributed mainly throughout the cytoplasm. It is principally known for its ability to hydrolyse DNA and is particularly active during the execution phase of apoptosis. It has previously been reported that the expression of DNase I is increased prior to the induction of apoptosis, with higher concentrations in the perinuclear space[29]. This is particularly true for cell types displaying rapid turnover rates, and ensures that the actual apoptotic event is rapid and efficient. Rapidly dividing cells exhibit low rates of apoptosis and so the virulent nature of these cells may explain the low DNase I staining with no particular areas of localisation.

In this report, the presence of an overlapped signal from the FITC and Cy3 probes indicates the colocalisation of DNase I and cofilin. Since we have previously reported that cofilin and DNase I do not bind to each other, we conclude that where ever they co-localise, they must be linked via actin. This can occur in vivo either due to the coincidence of a cofilinactin and actin-DNase I and/or the existence of cofilin- actin-DNase I ternary complex. Similar techniques have been used previously to demonstrate interactions between actin and myosin[30] and the formation of complexes between the ABPs a-actinin and Nspl1[31] as well as between insulin and its associated receptor[32]. 
Our native-PAGE data suggest that cofilin and DNase I bind more tightly to actin- DNase I and cofilin-actin binary complexes respectively than for actin alone. In other words, the ternary complex is more stable than either of the binary complexes. It is therefore noteworthy that in the dividing cell, there is relatively little co-localised cofilin and DNase I. Conversely the quiescent cell has a higher proportion of DNase I sequestered in a ternary complex. It is likely that the remainder of the labelled protein is associated with actin suggesting the action of an intracellular mechanism that allows the binary and ternary complexes to co-exist within the cell. The incidence of co-localisation is 6-fold higher in the non-dividing cell than in the dividing cell. The differences in co-localisation between the quiescent and dividing cells implies that DNase I may modulate the regulatory activity of cofilin during cell division.

Not only may cofilin and DNase I influence the binding to actin of each other, they may influence the binding of other ABPs, such as thymosin b4. We know that cofilin and thymosin b4 compete for binding to actin (Irina Dedova, personal communication). DNase I binding to actin inhibits the binding of thy$\operatorname{mosin} \beta 4$ [33] and in this case DNase I may be a mediator in the exchange of bound thymosin b4 on actin for cofilin. This process is outlined in Fig 4. We have demonstrated that the cofilin-actin-DNase I ternary complex is more stable than either binary complex. The mechanism of dissociation of DNase I from the ternary complex has not yet been determined, but probably involves phosphorylation or the binding of phosphoinositides [11].

DNase I affinity columns are widely used in the preparation of a number of muscle proteins including villin, actolinkin, destrin, actoseverin and radixin, which implies that these proteins also form ternary complexes involving actin-DNase I. Cofilin has also been shown to bind actin simultaneously with actin-interacting protein (Aip1). This coordinated binding of multiple ABPs to actin would allow finer control of the state of actin assembly.

In this report we have presented the first evidence supporting the co-existence of a cofilin-actinDNase I ternary complex with the cofilin-actin and actin-DNase I binary complexes in vivo. We propose that DNase I may be important in modulating the binding of other ABPs, e.g. cofilin and thymosin $\beta 4$.
This hypothesis is currently under investigation in vivo. Our observations introduce further regulatory aspects of $\mathrm{ABP}$ cascades in the control of cytoskeletal dynamics.

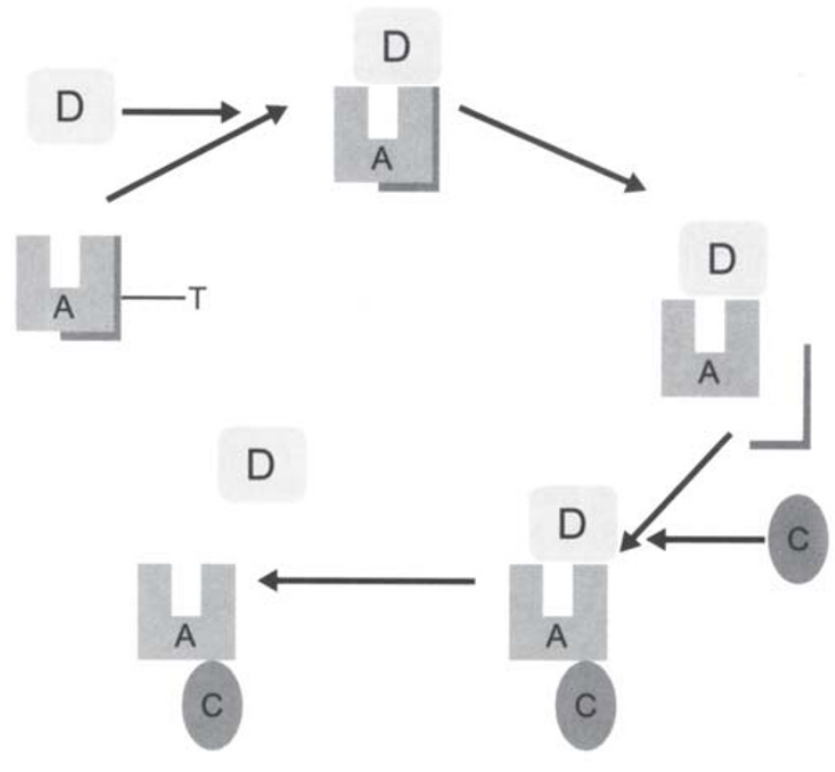

Fig 4. Illustration of the DNase I mediated exchange of thymosin $\beta 4$ for cofilin on actin. A-actin; T-thymosin $\beta$; DDNase I; C-cofilin.

\section{ACKNOWLEDGMENTS}

We would like to thank Dr. Neil Nosworthy for supplying his expertise with the native PAGE. This work was supported by grants from the Australian Research Council and the Ramaciotti Foundation.

\section{REFERENCES}

[1] dos Remedios CG, D Chhabra, M Tsubakihara, I Dedova, M Kekic, D Berry, NJ Nosworthy. Actin Binding Proteins and Regulation of Cytoskeletal Microfilaments. Physiological Reviews In Press.

[2] Goldschmidt-Clermont PJ, LM Machesky, SK Doberstein, TD Pollard. The mechanisms of interactions of human platelet cofilin with actin. J Cell Biol 1991; 113:1081-9.

[3] Teubner A, A Wegner. Kinetic evidence for a readily exchangeable nucleotide at the terminal subunit of the barbed ends of actin filaments. Biochemistry 1998; 37: 7532-8.

[4] Blanchoin L, TD Pollard. Interaction of actin monomers with Acacnthamoeba actophorin (ADF/cofilin) and profilin. J Biol Chem 1998; 273:25106-11.

[5] McGough A, B Pope, W Chiu, A Weeds. Cofilin changes the twist of F-actin: Implications for actin filament dynamics and cellular function. J Cell Biol 1997; 138:771-81.

[6] Bernstein BW, JR Bamburg. Tropomyosin binding to F- 
actin protects the F-actin from disassembly by brain actindepolymerizing factor (ADF). Cell Motil Cytoskel 1982; 2:1-8

[7] Maciver SK, HG Zot, TD Pollard. Characterization of actin filament severing by actophorin from Acanthamoeba castellanii. J Cell Biol 1991; 115:1611-20.

[8] Yonezawa N, E Nishida, H Sakai. pH control of actin polymerization by cofilin. J Biol Chem 1985; 260:14410-2.

[9] Morgan TE, RO Lockerbie, LS Minamide, MD Browning, JR Bamburg. Isolation and characterisation of a regulated form of actin depolymerizing factor. J Cell Biol 1993; 122:623-33.

[10] Arber S, FA Barbayannis, H Hanser, C Schneider, CA Stanyon, O Bernard, P Caroni. Regulation of actin dynamics through phosphorylation of cofilin by LIM-kinase. Nature 1998; 393:805-9.

[11] Yonezawa N, E Nishida, K Iida, I Yahara, H Sakai. Inhibition of the interactions of cofilin, destrin and deoxyribonuclease I with actin by phoshoinositides. J Biol Chem 1990; 265:8382-6.

[12] Falcone RAJ, LE Stern, CJ Kemp, CE Shin, CR Erwin, BW Warner. Apoptosis and the pattern of DNase I expression following massive small bowel resection. J Surg Res $1999 ; \mathbf{8 4}: 218-22$.

[13] Lazarides E, U Lindberg. Actin is a naturally occurring inhibitor of Deoxyribonuclease I. Proc Nat Acad Sci USA 1974; 71:4724-461.

[14] Weber A, CR Pennise, M Pring. DNase I increases the rate constant of depolymerizaton at the pointed (-) end of actin filaments. Biochemistry 1994; 33:4780-6.

[15] Kabsch W, HG Mannherz, D Suck, EF Pai, KC Holmes. Atomic structure of the actin: DNase I complex. Nature 1990; 347:37-44.

[16] Kekic M, NJ Nosworthy, I Dedova, C Collyer, CG dos Remedios. Regulation of the Cytoskeleton Assembly: a role for a Ternary complex of Actin with Two Actin-Binding Proteins. Resul Prob Cell Differ 2000; 32:165-79.

[17] Chhabra D, NJ Nosworthy, CG dos Remedios. The role of ATP, ADP and divalent cations in the formation of binary and ternary complexes of actin with cofilin and DNase I. Electrophoresis 2000; 21:3863-9.

[18] Nosworthy NJ, M Kekic, CG dos Remedios. The affinity of chick cofilin for actin increases when actin is complexed with DNase I: formation of a cofilin-actin-DNase I ternary complex. Proteomics 2001; 1:1513-8.

[19] Spudich JA, S Watt. The regulation of rabbit skeletal muscle contraction. I Biochemical studies of the interaction of tropomyosin-troponin complex with actin and the proteolytic fragments of myosin. J Biol Chem 1971; 246: 4866-71.

[20] Barden JA, CG dos Remedios. The environment of the high affinity cation binding site on actin and the separation between cation and ATP sites as revealed by proton
NMR and fluorescence spectroscopy. J Biochemistry 1984; 96:913-21.

[21] Houk W, K Ue. The measurement of actin concentration in solution: A comparison of methods. Analyt Biochemistry 1974; 62:66-74.

[22] Abe H, Endo, T., Yamamoto, K., Obinata, T. Sequence of cDNAs encoding actin depolymerizing factor and cofilin of embryonic chicken skeletal muscle: two functionally distinct actin-regulatory proteins exhibit high structural homology. Biochemistry 1990; 29:7420-5.

[23] Lindberg U. Molecular weight and amino acid composition of deoxyribonuclease I. Biochemistry 1967; 6:33542.

[24] Laemmli UK. Cleavage of structural proteins during the assembly of the head of bacteriophage T4. Nature 1970; 227:680-5.

[25]Svitkina TM, GG Borisy. Arp2/3 complex and actin depolymerizing factor cofilin in dendritic organization and treadmilling of actin filament array in lamellipodia. $\mathrm{J}$ Cell Biol 1999; 145:1009-26.

[26] Nagaoka R, H Abe, K Kusano, T Obinata. Concentration of cofilin, a small actin binding protein, at the cleavage furrow during cytokinesis. Cell Motil Cytoskel 1995b; 30:1-7.

[27] Nagaoka R, K Kusano, H Abe, T Obinata. Effects of cofilin on actin filamentous structures in cultured muscle cells - Intracellular regulation of cofilin action. J Cell Sci 1995a; 108:581-93.

[28] Maupin P, TD Pollard. Arrangement of actin filaments and myosin-like filaments in the contractile ring and of actin-like filaments in the mitotic spindle of dividing HeLa cells. J Ultrastruct Mol Struct Res 1986; 94:92103.

[29] Polzar B, S Zanotti, H Stephan, F Rauch, MC Peitsch, M Irmler, et al. Distribution of deoxyribonuclease I in rat tissues and its correlation to cellular turnover rates and apoptosis (programmed cell death). Eur J Cell Biol 1994; 64:200-10.

[30] Mandato CA, WM Bement. Contraction and polymerization cooperate to assemble and close actomyosin rings around Xenopus oocyte wounds. J Cell Biol 2001; 154: 785-97.

[31] Geisler JG, RJ Palmer, LJ Stubbs, ML Mucenski. Nspl1, a new Z-band-associated protein. J Muscle Res Cell Motil 1999; 20:661-8.

[32] Karagiannis SN, RH King, PK Thomas. Colocalisation of insulin and IGF-1 receptors in cultured rat sensory and sympathetic ganglion cells. J Anat 1997; 191:431-40.

[33] Ballweber E, E Hannappel, T Huff, HG Mannherz. Mapping the binding site of thymosin b4 on actin by competition with G-actin binding proteins indicates negative co-operativity between binding sites located on opposite subdomains of actin. Biochem J 1997; 327:787-93. 